'Departamento de Cirugía Vascular y Endovascular, Pontificia Universidad Católica de Chile. Santiago, Chile. 2Departamento de Hematología, Pontificia Universidad Católica de Chile. Santiago, Chile.

${ }^{3}$ Departamento de Medicina

Interna, Pontificia Universidad Católica de Chile. Santiago, Chile.

${ }^{4}$ Laboratorio de Hemostasia, Pontificia Universidad Católica de Chile. Santiago, Chile. aTecnólogo Médico.

No hubo fuente de apoyo financiero.

Recibido el 5 de marzo de 2014 aceptado el 15 de octubre de 2014

Correspondencia a: Dr. Leopoldo Mariné Massa Apoquindo 3990, oficina 601 , Las Condes, Santiago. marinepolo@yahoo.com

\section{Correlación de valores de TTPa con anti factor Xa para establecer rango terapéutico en tratamiento anticoagulante con heparina sódica}

\author{
LEOPOLDO MARINÉ1, GONZALO SÁNCHEZ ${ }^{1}$, JOSÉ FRANCISCO VARGAS ${ }^{1}$, \\ PAMELA ZÚÑIGA ${ }^{2}$, ANDRÉS AIZMAN ${ }^{3}$, RENATO MERTENS ${ }^{1}$, \\ MICHEL BERGOEING ${ }^{1}$, BLANCA MUÑOZ ${ }^{4, a}$

\section{Correlation between aPTT and antifactor $\mathrm{Xa}$ to determine therapeutic ranges for unfractionated heparin}

Background: The therapeutic range (TR) of activated partial thromboplastin time (aPTT) for unfractionated heparin (UFH) dosing was established in the 1970 decade. Since then aPTT determination has changed. Current TR may be sub or supra-therapeutic depending on the reagents of the test, and therefore, responsible for complications of therapy. Aim: To establish the TR for UFH dosing in our institution using antifactor Xa analysis as reference standard. Material and Methods: After obtaining an informed consent, 43 blood samples were obtained for aPTT determination and antifactor Xa assay in 23 patients treated with intravenous UFH. Samples were processed at Emergency and Hemostasis Labs. We excluded patients receiving other anticoagulants, with thrombophilia, pregnancy or liver disease. Results: Mean aPTT values in the Hemostasis and Emergency labs were $57.1 \pm 18.9$ and $56.6 \pm 18.3$ seconds, respectively $(p=0.77)$. The squared correlation coefficients between aPTT and antifactor Xa at hemostasis and emergency labs were $R 20.5$ and 0.45 respectively, $p<0.001$. Using a linear regression analysis, therapeutic aPTT range values in our laboratory were established between 50 and 80 seconds, corresponding to antifactor Xa values of 0.3 to $0.7 \mathrm{IU} / \mathrm{mL}$. Conclusions: According to current recommendations, validation of aPTT determination with reference techniques should be done in every institution.

(Rev Med Chile 2014; 142: 1392-1397)

Key words: Blood Coagulation; Factor Xa; Heparin; Nomograms; Partial Thromboplastin Time.
4

1 tratamiento anticoagulante con heparina no fraccionada (HNF) tiene sus mayores beneficios cuando se logra el nivel de anticoagulación deseado. Su administración en dosis inadecuadas es responsable de hemorragias ante tratamiento excesivo ${ }^{1}$ o progresión de la trombosis si es sub-terapéutica ${ }^{2}$. El éxito del tratamiento anticoagulante dependerá entonces de la exactitud y estabilidad de la dosis indicada basada en una adecuada monitorización de los niveles de anticoagulación ${ }^{2}$.

La dosificación de la HNF en la práctica clínica se realiza a partir de la monitorización con valores de tromboplastina parcial activada (TTPa), ajustándose éstas de acuerdo a diferentes esquemas o nomogramas ${ }^{3}$. El objetivo es alcanzar un rango 
de anticoagulación efectiva o rango terapéutico (RT) de TTPa que permita el efecto terapéutico deseado y sea seguro ${ }^{2}$.

La determinación de TTPa es un proceso automatizado, de bajo costo y permanentemente disponible en todos los centros hospitalarios. La prueba requiere de reactivos químicos y de un coagulómetro que establece el tiempo que demora la muestra en producir fibrina.

El TTPa presenta limitaciones al no tener un sistema de estandarización como es el INR para el tiempo de protrombina. Por otro lado, cambios en los componentes de la prueba modifican los valores esperados de TTPa y por tanto, el rango terapéutico, aunque el nomograma sea utilizado en forma correcta, con resultados clínicos que pueden ser desfavorables al no ajustarse los valores de TTPa a la realidad del efecto de la droga. A modo de ejemplo, una determinación de valores de TTPa de 60 a 85 segundos de RT pueden corresponder a valores sub-terapéuticos, terapéuticos o supraterapéuticos dependiendo de cuáles componentes fueron utilizados en la prueba de TTPa ${ }^{4}$.

Considerando lo anterior, para mejorar la calidad y seguridad del tratamiento con HNF, la recomendación vigente es correlacionar los valores de TTPa con otro examen que tenga una mayor sensibilidad y especificidad previo a su uso en la monitorización de la dosificación de heparina. Aunque no existen estudios prospectivos con distribución aleatoria, la determinación de niveles de heparina medidos en plasma bajo técnica de titulación de protamina y la medición de valores de anti factor Xa han sido utilizadas como exámenes de referencia para el TTPa en múltiples publicaciones $^{3-13}$, correspondiendo el RT para éstas de 0,2 a $0,4 \mathrm{UI} / \mathrm{ml}$ para la primera y de 0,3 a $0,7 \mathrm{UI} / \mathrm{ml}$ para la segunda. Es importante señalar que este tipo de rectificación debe ser realizada en cada laboratorio y debe repetirse cada vez que se cambien los reactivos o el coagulómetro.

El presente trabajo tiene por objetivo establecer el RT de TTPa para pacientes en tratamiento con HNF en nuestra institución, al correlacionar los valores de TTPa con la determinación de anti factor Xa como referencia.

\section{Material y Método}

Se realizó un estudio de test diagnóstico, con aprobación por el Comité de Ética Clínica de la
Pontificia Universidad Católica de Chile (número 12-211). El objetivo primario es establecer el RT para HNF en nuestra institución, mediante la confección de una curva de correlación entre los valores simultáneos de TTPa y de anti factor Xa. El diseño metodológico contempla como objetivo secundario establecer la existencia de diferencias entre los valores de TTPa resultantes del procesamiento de la muestra con centrifugación simple o doble.

Se reclutaron pacientes entre septiembre de 2012 y enero de 2013 que se encontraban en tratamiento endovenoso con HNF, con medición periódica de TTPa, que cumplieron los criterios de inclusión y exclusión (Tabla 1) y que firmaron el consentimiento informado.

A los pacientes seleccionados se tomaron muestras de sangre por la enfermera según las normas de SINFEX (Sistema de Información de Exámenes de la Red de Salud UC), en el momento correspondiente a su control habitual de TTPA, siguiendo la indicación del médico de la unidad en que se encontraba hospitalizado. Participaron en el estudio pacientes hospitalizados bajo el cuidado de 3 grupos de médicos tratantes: Neurología, Cardiología y Cirugía Vascular.

A partir de la muestra se prepararon dos tubos BD Vacutainer ${ }^{\circledR}$ tapa celeste (BD Franklin Lakes, NJ, EEUU), los cuales contenían 0,5 cc de citrato que se completaron con $4,5 \mathrm{cc}$ de sangre cada uno. Uno de estos tubos se envió al Laboratorio de Urgencia, como es la práctica para la determinación de TTPa en nuestro hospital; el otro tubo fue enviado al Laboratorio de Hemostasia. Todas las muestras, luego de ser tomadas, fueron trasladadas de inmediato a estos laboratorios por uno de los investigadores y fueron procesadas en las horas siguientes.

En el Laboratorio de Urgencia se realizó centrifugación simple de la muestra a temperatura ambiente y luego la determinación del TTPa con reactivo APTT-SP (Instrumentation Laboratory, Lexington, Massachusetts, EEUU), utilizándose un coagulómetro ACL-TOP (Instrumentation Laboratory, Lexington, Massachusetts, EEUU) para completar la determinación del TTPa. En el Laboratorio de Hemostasia, se sometió la muestra a doble centrifugación: por 10 min a 2.000 g y luego por 5 min a $1.000 \mathrm{~g}$, a temperatura ambiente. Las alícuotas se separaron para la determinación de TTPa utilizando los mismos reactivos y tam- 
Tabla 1. Criterios de inclusión y exclusión

\begin{tabular}{|ll}
\hline Criterios de Inclusión & Criterios de Exclusión \\
- Paciente hospitalizado & - Tratamiento concomitante con Heparina de bajo peso \\
- Diagnósticos: & molecular y/o Tratamiento anticoagulante oral \\
Trombosis venosa profunda o patología arterial que re- & - Uso de inhibidores directos de la trombina \\
quiera terapia con heparina no fraccionada en infusión & - Elevación conocida del factor IV plaquetario \\
continua & - Déficit conocido de Vitamina K \\
- Paciente debe tener un Tiempo de Protrombina - INR y & - Trombofilia congénita o adquirida conocida \\
un TTPa basal normal & - Síndrome antifosfolípidos conocido \\
& - Contaminación de la muestra con heparina \\
& - Embarazo \\
& - Enfermedades hepáticas conocidas que alteren las prue- \\
& bas de coagulación \\
& - Valores de TTPa mayores de 120 segundos
\end{tabular}

bién un coagulómetro idéntico modelo ACL-TOP (Instrumentation Laboratory, Lexington, Massachusetts, EEUU) y la determinación de anti factor Xa por técnica de Biophen Heparin 6, método cromogénico de determinación.

Para cada muestra se obtuvo tres resultados: un valor de TTPA del Laboratorio de Urgencia (utilizado por su equipo tratante para guiar el tratamiento), un valor de TTPA del Laboratorio de Hemostasia y un valor de anti factor Xa, requiriéndose contar con resultados válidos en estos tres exámenes para que la muestra fuera incluida dentro del estudio (Tabla 1 ).

En el análisis estadístico se utilizó el programa GraphPad 5.0 (GraphPad Software, Inc.). Se construyeron curvas de regresión a partir de puntos determinados por el valor de TTPa de cada muestra y su correspondiente valor de anti factor Xa. Se hizo análisis de regresión lineal para los valores de TTPA obtenidos en el Laboratorio de Urgencia y en el Laboratorio de Hemostasia con un intervalo de confianza de $95 \%$, obteniéndose el RT. Los promedios fueron comparados utilizando test de Student pareado.

\section{Resultados}

Entre septiembre de 2012 y enero de 2013 se reclutaron 23 pacientes y se recolectaron 43 muestras de sangre. Hubo 3 muestras que no cumplieron con los criterios de la Tabla 1 por lo que fueron excluidas.
La correlación entre anti factor Xa y los valores de TTPA obtenidos en cada uno de los laboratorios, fue estadísticamente significativa: $\mathrm{R}^{2}$ del Laboratorio de Hemostasia: 0,4986 ( $<<0,0001) ; \mathrm{R}^{2}$ del Laboratorio de Urgencia: 0,4466 ( $\mathrm{p}<0,0001)$. Para obtener la correlación lineal del TTPA fue necesario eliminar dos valores de TTPA que fueron confirmados como "outliers" significativos ${ }^{14}$ (Figuras 1 y 2).

Ambas curvas de regresión lineal fueron concordantes en inferir que los valores terapéuticos de TTPa para nuestro laboratorio estarían entre los 50 y los 80 segundos, correspondiendo a valores de anti factor Xa de 0,3 a $0,7 \mathrm{UI} / \mathrm{mL}$ y siendo el $\mathrm{RT}$ en el que deben basarse los nomogramas para subir o bajar las dosis de HNF.

El promedio de valor de TTPa obtenido fue de 57,1 $\pm 18,9$ segundos para el Laboratorio de Hemostasia y de 56,6 $\pm 18,3$ segundos para el Laboratorio de Urgencia, diferencia que no es estadísticamente significativa $(\mathrm{p}=0,77)$.

\section{Discusión}

Las dosis terapéuticas de HNF utilizadas en la práctica clínica se establecieron a partir de un estudio observacional de la década 1970-79², determinándose posteriormente que el RT se alcanza cuando los valores de TTPa son 1,5 a 2,5 veces el valor de control basal del laboratorio ${ }^{2,5,15}$. Aunque la evidencia que lo apoya es débil y no existe confirmación por estudios de distribución 


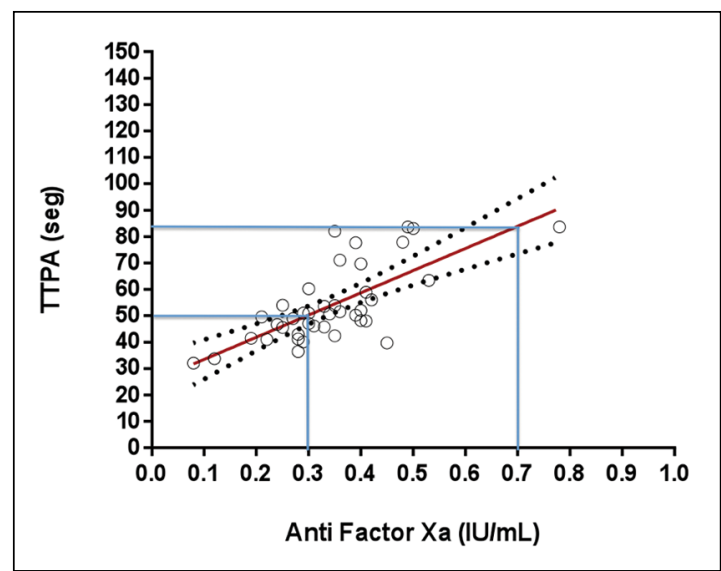

Figura 1. Correlación lineal entre valores de Anti Factor Xa y de TTPa obtenidos en el Laboratorio de Hemostasia.

aleaoria ${ }^{3}$, en la práctica clínica actual esta fórmula se utiliza frecuentemente para el cálculo de RT en pacientes bajo anticoagulación con HNF y que luego son incorporados a nomogramas de manejo que descansan en la confiabilidad de la determinación del TTPa.

La determinación de TTPa en el laboratorio se afecta al modificar uno o ambos componentes esenciales de la prueba: los reactivos o el instrumento de medición. Dentro de los reactivos, además del calcio participan distintos tipos de activadores de contacto (kaolin, silica micronizada, ácido elágico) y fosfolípidos (cefalinas) ${ }^{8}$. A la fecha, existen más de 300 reactivos diferentes ${ }^{4} \mathrm{y}$ diversos tipos de instrumentos de medición automatizada o coagulómetros ${ }^{8}$. Es importante destacar el hecho que los componentes de la prueba de TTPa utilizados en los estudios de validación inicial antes mencionados ${ }^{2,5,15}$, han cambiado en múltiples ocasiones desde entonces. Las variadas posibilidades de combinación de los componentes de la prueba determinan no sólo eventuales diferencias en los valores de TTPa y por tanto, también en el RT ${ }^{7,15-17}$.

Desde hace unos años se ha advertido que los valores de TTPa obtenidos con los coagulómetros y reactivos actuales son responsables de fluctuación en el RT, los cuales pueden extenderse entre los 43,6 y los 165,2 segundos ${ }^{8}$, los que corresponden a 1,6-2,7 a 3,7-6,2 veces el valor de control basal $^{3}$, valores muy distantes del RT histórico (1,5 a $2,5)$. Desde otro punto de vista, un mismo paciente puede presentar diversos valores de RT de TTPa

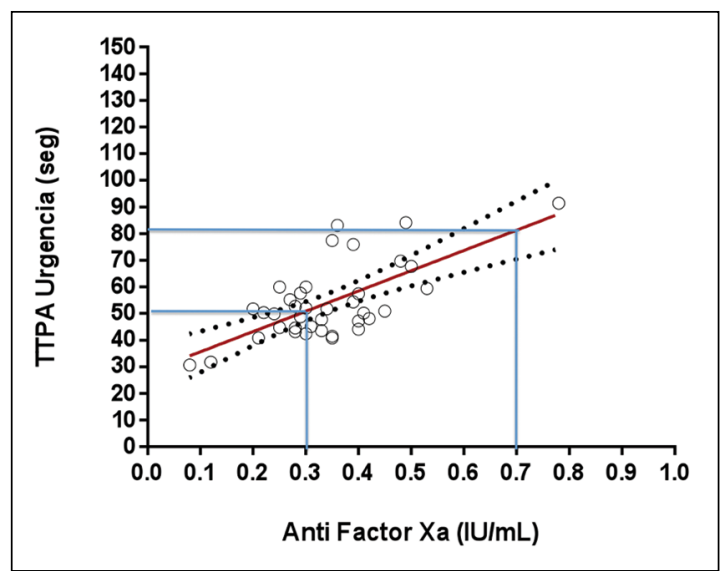

Figura 2. Correlación lineal entre valores de Anti Factor Xa y de TTPa obtenidos en el Laboratorio de Urgencia.

según los reactivos o coagulómetros utilizados en su determinación ${ }^{8}$. Esta gran variabilidad obliga a que la prueba de TTPa sea validada en cada institución utilizando exámenes de laboratorio de mayor especificidad y sensibilidad que el TTPa.

La determinación de niveles de heparina medidos en plasma bajo técnica de titulación de protamina y la medición de valores de anti factor Xa son utilizadas como pruebas de referencia debido a mayor exactitud, menor variabilidad que el TTPa por menor influencia por factores biológicos (anticoagulante lúpico, factor VIII elevado, patologías hepáticas) o factores extrínsecos (anticoagulantes orales, concentración de citrato de sodio en el tubo de la muestra) ${ }^{9}$. En consideración de estas ventajas podría plantearse su uso rutinario en la monitorización de HNF, pero esto no ha sido posible debido a limitaciones en disponibilidad de acceso, complejidad técnica y alto costo. A pesar de esto, algunos centros las han incorporado en sus esquemas de monitorización ${ }^{9-11,18}$.

Considerando la evidencia disponible, la recomendación vigente de sociedades como el American College of Chest Physicians ${ }^{3}$ y otras ${ }^{6,19}$, es corregir los valores de RT por TTPa con métodos de determinación más exactos en cada laboratorio y cada vez que se modifique la técnica de la prueba de TTPa. Se sugiere confeccionar curvas de correlación de $\mathrm{TTPa}^{14}$, con métodos como la titulación de protamina o la medición de anti factor $\mathrm{Xa}^{7,8}$, siendo necesario comparar al menos de $30^{7,8}$ a $40^{14}$ muestras. 
En cumplimiento de estas recomendaciones internacionales, quisimos establecer el RT de TTPa en nuestra institución al correlacionar valores de TTPa con sus correspondientes valores de anti factor Xa en 40 muestras. Las muestras fueron tomadas de pacientes portadores de patología arterial o venosa con indicación de anticoagulación terapéutica con HNF, siendo excluidos los pacientes que presentaban condiciones médicas que pudiesen interferir adicionalmente los valores obtenidos de TTPa, tales como embarazo, trombofilias congénitas o adquiridas, patologías hepáticas que alteren las pruebas de coagulación y otras que se describen en la Tabla 1. Dentro de las pruebas de referencia, nuestra institución cuenta con la determinación de anti factor Xa en forma rutinaria, prueba que mide la capacidad del complejo Hep-ATIII de inhibir al factor Xa. La existencia de centros hospitalarios que no se disponen de determinación de anti factor Xa limita el cumplimiento masivo de esta recomendación, siendo posible recurrir a la asistencia de un laboratorio externo para efectuar la correlación.

A partir de los análisis finales de las muestras se pudo establecer que valores de TTPa entre 50 y 80 segundos corresponden al RT de HNF para nuestra institución, como se puede inferir de las Figuras 1 y 2, cuyos RT son casi los mismos. Secundariamente, pudimos además comprobar que la determinación de TTPa no varía si la muestra es sometida a centrifugación simple o doble. Secundariamente, al realizar determinación de TTPa en dos laboratorios distintos pero utilizando igual técnica, pudimos además comprobar que no existe diferencia significativa en sus valores si la muestra es sometida a centrifugación simple o doble. Con la información obtenida, el TTPa podrá continuar siendo el método de monitorización de elección para HNF en nuestra institución, pero de forma más exacta y segura. Además será posible confeccionar nomogramas locales más precisos y eficientes, de acuerdo a valores de RT reales, lo que permitirá finalmente optimizar el tratamiento anticoagulante $y$, a su vez, hacerlo más seguro.

Una posible limitación de este estudio es el menor número de muestras con TPPa altos respecto a los menores de 80 segundos. Debe considerarse que con el uso HNF en dosis altas, existe mayor posibilidad de que el valor de TTPa sea indeterminable (mayor de 120 segundos), valor que constituye un criterio de exclusión en este estudio. Similar limitación en número de valores altos de TTPa en las curvas de correlación han sido observadas en otros estudios ${ }^{7,11}$.

\section{Conclusión}

Los resultados obtenidos de este estudio permiten establecer que el RT de HNF en nuestra institución corresponde a valores de TTPa entre 50 y 80 segundos.

La información de valores de TTPa obtenida no es extrapolable a otros centros hospitalarios, considerando que las distintas combinaciones de reactivos y coagulómetros pueden determinar que sus valores de TTPa en RT sean diferentes a los presentados. Cada centro deberá realizar sus propios estudios de test diagnósticos.

\section{Referencias}

1. Morabia A. Heparin doses and major bleedings. Lancet 1986; 1 (8492): 1278-9.

2. Basu D, Gallus A, Hirsh J, Cade J. A prospective study of the value of monitoring heparin treatment with the activated partial thromboplastin time. N Engl J Med 1972; 287 (7): 324-7.

3. García DA, Baglin TP, Weitz JI, Samama MM; American College of Chest Physicians. Parenteral anticoagulants: Antithrombotic Therapy and Prevention of Thrombosis, 9th ed: American College of Chest Physicians EvidenceBased Clinical Practice Guidelines. Chest 2012; 141 (2 Suppl): e24S-43S.

4. Raschke R, Hirsh J, Guidry JR. Suboptimal Monitoring and Dosing of Unfractionated Heparin in Comparative Studies with Low-Molecular-Weight Heparin. Ann Intern Med 2003; 138 (9): 720-3.

5. Hirsh J, Fuster V. Guide to anticoagulant therapy. Part 1: Heparin. American Heart Association. Circulation 1994; 89 (3): 1449-68.

6. Olson JD, Arkin CF, Brandt JT, Cunningham MT, Giles A, Koepke JA, et al. College of American Pathologists Conference XXXI on laboratory monitoring of anticoagulant therapy: laboratory monitoring of unfractionated heparin therapy. Arch Pathol Lab Med 1998; 122 (9): 782-98.

7. Brill-Edwards P, Ginsberg JS, Johnston M, Hirsh J. Establishing a therapeutic range for heparin therapy. Ann Intern Med 1993; 119 (2): 104-9.

8. Bates SM, Weitz JI, Johnston M, Hirsh J, Ginsberg JS. Use 
of a Fixed Activated Partial Thromboplastin Time Ratio to Establish a Therapeutic Rangefor Unfractionated Heparin. Arch Intern Med 2001; 161 (3): 385-91.

9. Guervil DJ, Rosenberg AF, Winterstein AG, Harris NS, Johns TE, Zumberg MS. Activated partial thromboplastin time versus antifactor Xa heparin assay in monitoring unfractionated heparin by continuous intravenous infusion. Ann Pharmacother 2011; 45 (7-8): 861-8.

10. Vandiver JW, Vondracek TG. Antifactor Xa levels versus activated partial thromboplastin time for monitoring unfractionated heparin. Pharmacotherapy 2012; 32 (6): 546-58.

11. Rosenberg AF, Zumberg M, Taylor L, LeClaire A, Harris $\mathrm{N}$. The use of anti-Xa assay to monitor intravenous unfractionated heparin therapy. J Pharm Pract 2010; 23 (3): 210-6.

12. Levine MN, Hirsh J, Gent M, Turpie AG, Cruickshank $\mathrm{M}$, Weitz J, et al. A randomized trial comparing activated thromboplastin time with heparin assay in patients with acute venous thromboembolism requiring large daily doses of heparin. Arch Intern Med 1994; 154 (1): 49-56.

13. Hirsh J, Warkentin TE, Shaughnessy SG, Anand SS, Halperin JL, Raschke R, et al. Heparin and low-molecularweight heparin: mechanisms of action, pharmacokinetics, dosing, monitoring, efficacy, and safety. Chest 2001; 119 (1 Suppl): 64S-94S.

14. Method Comparison and Bias Estimation Using Patient Samples; Approved Guideline-Second Edition. NCCLS document EP9-A2 (ISBN 1-56238-472-4). NCCLS,
940 West Valley Road, Suite 1400, Wayne, Pennsylvania 19087-1898 USA. NCCLS document EP9-A2 2002; 22 (19): 1-56.

15. Gawoski J, Arkin CF, Bovill T, Brandt J, Rock WA Jr, Triplett DA. The effects of heparin on the activated partial thromboplastin time of the College of American Pathologists survey specimens: responsiveness, precision, and sample effects. Arch Pathol Lab Med 1987; 111 (9): 785-90.

16. Stevenson KJ, Eaton AC, Curry A, Thomson JM, Poller L. The reliability of activated partial thromboplastin time methods and the relationship to lipid composition and ultrastructure. Thromb Haemost 1986; 55 (2): 250-8.

17. Bjornsson TD, Nash PV. Variability in heparin sensitivity of APTT reagents. Am J Clin Pathol 1986; 86 (2): 199204.

18. Smith ML, Wheeler KE. Weight-based heparin protocol using antifactor Xa monitoring. Am J Health Syst Pharm 2010; 67 (5): 371-4.

19. Greaves M. Control of Anticoagulation Subcommittee of the Scientific and Standardization Committee of the International Society of Thrombosis and Haemostasis. Limitations of the laboratory monitoring of heparin therapy. Scientific and Standardization Committee Communications: on behalf of the Control of Anticoagulation Subcommittee of the Scientific and Standardization Committee of the International Society of Thrombosis and Haemostasis. Thromb Haemost 2002; 87 (1): 163-4. 